\title{
Preliminary Definitions for Sacroiliac Joint Pathologies in the OMERACT Juvenile Idiopathic Arthritis Magnetic Resonance Imaging Score (OMERACT JAMRIS-SIJ)
}

Tarimobo M. Otobo (D), Philip G. Conaghan, Walter P. Maksymowych, Desiree van der Heijde, Pamela Weiss (D), Iwona Sudol-Szopinska, Nele Herregods, Jacob L. Jaremko, Arthur B. Meyers, Dax Rumsey, Emilio C. Inarejos, Eva Kirkhus, Jennifer Stimec, Jyoti Panwar ${ }^{\mathbb{D}}$, Kevin Thorpe, Lennart Jans, M.A. van Rossum (D), Mirkamal Tolend, Manuela Perez, Nikolay Tzaribachev, Pulukool Sandhya, Shirley Tse, Appenzeller Simone, Vimarsha G. Swami, Zahi Touma, Robert Lambert ${ }^{(}$, and Andrea S. Doria

ABSTRACT. Objective. To develop definitions for the assessment of magnetic resonance imaging (MRI) pathologies of the sacroiliac joints (SIJ) in juvenile idiopathic arthritis.

Methods. An Outcome Measures in Rheumatology (OMERACT) consensus-driven methodology consisting of iterative surveys and focus group meetings within an international group of rheumatologists and radiologists.

Results. Two domains, inflammation and structural, were identified. Definitions for bone marrow edema, joint space inflammation, capsulitis, and enthesitis were derived for joint inflammation; sclerosis, erosion, fatty lesion, and ankylosis were defined for assessing structural joint changes.

Conclusion. Preliminary consensus-driven definitions for inflammation and structural elements have been derived, underpinning the ongoing development of the OMERACT Juvenile Arthritis MRI SIJ scoring system (OMERACT JAMRIS-SIJ). (First Release May 15 2019; J Rheumatol 2019;46:1192-7; doi:10.3899/jrheum.181115)

Key Indexing Terms:

OMERACT MAGNETIC RESONANCE IMAGING JUVENILE IDIOPATHIC ARTHRITIS OUTCOME ASSESSMENT

SACROILIITIS

From the Institute of Medical Sciences, Faculty of Medicine, University of Toronto; Department of Diagnostic Imaging, The Hospital for Sick Children; Department of Translational Medicine, SickKids Research Institute, Peter Gilgan Center for Research and Learning; Dalla Lana School of Public Health, University of Toronto; Division of Rheumatology, The Hospital for Sick Children, University of Toronto; Department of Medical Imaging, University of Toronto; Department of Rheumatology, Center for Prognosis Studies in Rheumatologic Diseases, Toronto Western Hospital, Toronto, Ontario; Department of Rheumatology, University of Alberta; Department of Radiology and Diagnostic Imaging, University of Alberta; Division of Pediatric Rheumatology, Department of Pediatrics, University of Alberta, Edmonton, Alberta, Canada; Leeds Institute of Rheumatic and Musculoskeletal Medicine, University of Leeds; UK National Institute for Health Research (NIHR) Leeds Biomedical Research Centre, Leeds Teaching Hospitals National Health Service (NHS) Trust, Leeds, UK; Department of Rheumatology, Leiden University Medical Center, Leiden; Reade I Emma Children's Hospital/Academic Medical Center, Amsterdam, the Netherlands; University of Pennsylvania Perelman School of Medicine, Division of Rheumatology, Children's Hospital of Philadelphia and Departments of Pediatric and Epidemiology, Philadelphia, Pennsylvania; Department of Radiology, Nemours Children's Hospital and Health System, Orlando, Florida, USA; Department of Radiology, National Institute of Geriatrics, Rheumatology and Rehabilitation, Warsaw, Poland; Department of Radiology and Medical Imaging, Ghent University Hospital, Ghent, Belgium; Department of Radiology, Hospital Sant Joan de Deu, Barcelona, Spain; Department of Radiology, Rikshospitalet, Oslo University Hospital, Oslo, Norway; Pediatric Rheumatology Research Institute, Bad Bramstedt, Germany;
Department of Clinical Immunology and Rheumatology, Christian Medical College, Vellore, India; State University of Campina-UNICAMP, Department of Internal Medicine, Cidade Universitaria, Sao Paulo, Brazil.

P.G. Conaghan is funded in part by the NIHR Leeds Biomedical Research Centre. The views expressed are those of the authors and not necessarily those of the NHS, the NIHR, or the UK Department of Health.

T.M. Otobo, MD, MSc, BMedSc, Institute of Medical Sciences, Faculty of Medicine, University of Toronto, and Department of Diagnostic Imaging, The Hospital for Sick Children, and Department of Translational Medicine, SickKids Research Institute, Peter Gilgan Center for Research and Learning; P.G. Conaghan, PhD, MB BS, Leeds Institute of Rheumatic and Musculoskeletal Medicine, University of Leeds, and NIHR Leeds Biomedical Research Centre, Leeds Teaching Hospitals NHS Trust; W.P. Maksymowych, PhD, MB BS, FRACP, FRCP, Department of Rheumatology, University of Alberta; D. van der Heijde, $M D, P h D$, Department of Rheumatology, Leiden University Medical Center; P. Weiss, MD, MSCE, University of Pennsylvania Perelman School of Medicine, Division of Rheumatology, Children's Hospital of Philadelphia and Departments of Pediatric and Epidemiology; I. Sudol-Szopinska, MD, PhD, Department of Radiology, National Institute of Geriatrics, Rheumatology and Rehabilitation; N. Herregods, MD, PhD, Department of Radiology and Medical Imaging, Ghent University Hospital; J.L. Jaremko, MD, PhD, FRCPC, Department of Radiology and Diagnostic Imaging, University of Alberta; A.B. Meyers, MD, Department of Radiology, Nemours Children's Hospital and Health System; D. Rumsey, MD, FRCPC, Division of Pediatric Rheumatology, Department of Pediatrics, University of Alberta; E.C. Inarejos, $M D$, 
Juvenile idiopathic arthritis (JIA) is a chronic inflammatory disease that affects joint function, resulting in significant effects on quality of life by limiting physical and psychosocial integration ${ }^{1}$. JIA has an estimated worldwide prevalence of 0.07 to 4.01 cases and an incidence rate of 0.008 to 0.226 cases per 1000 children per year, respectively, highlighting the global disease burden and the need for new clinical trials for JIA management ${ }^{2}$.

Magnetic resonance imaging (MRI) has been reported to be sensitive in detecting early inflammatory changes in the sacroiliac joints (SIJ) in JIA long before radiography, thus allowing for intervention before the onset of irreversible joint damage $^{3}$. Clinical assessment of the SIJ is limited by its location and anatomy ${ }^{4}$. The quantification of interval change of pediatric SIJ using an MRI-based scoring method may serve as an important objective outcome measure ${ }^{5}$. The new JIA Clinical Core Set recently endorsed at the OMERACT 2018 conference includes joint inflammation and damage domains as variables in the middle circle, recommending these as important measurement domains in JIA clinical trials. Improving these outcome measures calls for an international, multidisciplinary effort toward development and validation of suitable and specific measurement instruments to quantify disease and assess treatment effects in JIA.

A recent systematic review by Swami, et $a l^{6}$ of existing MRI-based SIJ scoring systems reported the lack of established MRI instruments for assessment of SIJ in the pediatric

Department of Radiology, Hospital Sant Joan de Deu; E. Kirkhus, MD, PhD, Department of Radiology, Rikshospitalet, Oslo University Hospital; J. Stimec, MD, FRCPC, Department of Diagnostic Imaging, The Hospital for Sick Children; J. Panwar, MD, Department of Diagnostic Imaging, The Hospital for Sick Children; K. Thorpe, BMath, MMath, Dalla Lana School of Public Health, University of Toronto; L. Jans, MD, PhD. Department of Radiology and Medical Imaging, Ghent University Hospital; M.A. van Rossum, MD, PhD, Reade I Emma Children's Hospital/Academic Medical Center; M. Tolend, BSc, Institute of Medical Sciences, Faculty of Medicine, University of Toronto, and Department of Diagnostic Imaging, The Hospital for Sick Children, and Department of Translational Medicine, SickKids Research Institute, Peter Gilgan Center for Research and Learning; M. Perez, MD, Department of Diagnostic Imaging, The Hospital for Sick Children; N. Tzaribachev, MD, Pediatric Rheumatology Research Institute; P. Sandhya, MD, Department of Clinical Immunology and Rheumatology, Christian Medical College; S. Tse, MD, FRCPC, Division of Rheumatology, The Hospital for Sick Children, University of Toronto; A. Simone, MD, PhD, State University of Campina-UNICAMP, Department of Internal Medicine, Cidade Universitaria; V.G. Swami, MD, BSc, Department of Medical Imaging, University of Toronto; Z. Touma, MD, PhD, FACP, FACR, Department of Rheumatology, Center for Prognosis Studies in Rheumatologic Diseases, Toronto Western Hospital; R. Lambert, MB BCh, FRCR, FRCPC,

Department of Radiology and Diagnostic Imaging, University of Alberta; A.S. Doria, MD, PhD, MSc, Institute of Medical Sciences, Faculty of Medicine, University of Toronto, and Department of Diagnostic Imaging, The Hospital for Sick Children, and Leeds Institute of Rheumatic and Musculoskeletal Medicine, University of Leeds, and NIHR Leeds Biomedical Research Centre, Leeds Teaching Hospitals NHS Trust, and Department of Medical Imaging, University of Toronto.

Address correspondence to Dr. A.S. Doria, Department of Diagnostic Imaging, The Hospital for Sick Children, 555 University Ave., Toronto, Ontario M5G 1X8, Canada.E-mail: andrea.doria@sickkids.ca

Accepted for publication January 30, 2019. population. Conversely, SIJ MRI scoring systems are well developed and validated in adults $7,8,9,10$. Current attempts to extrapolate adult SIJ MRI scoring systems into the pediatric group reported significant feasibility and reliability, but the construct validity remains a limitation, although clinical assessment of disease activity and damage also remains problematic in the pediatric population ${ }^{11,12}$. Given the lack of standards for normality and pathology in growing SIJ, there is an urgent need for the development and validation of a pediatric-specific MRI scoring system for $\mathrm{SIJ}^{5}$. We used the Outcome Measures in Rheumatology (OMERACT) consensus-driven methodology for iteratively developing definitions as a prelude to the development of an MRI-based scoring system for the assessment of SIJ in JIA, and as a first step we aimed to decide on the main domains and elementary pathology definitions of the MRI-based scoring system.

\section{MATERIALS AND METHODS}

Conceptual framework of the OMERACT JAMRIS-SIJ. The OMERACT JAMRI Working Group developed the Juvenile Arthritis MRI SIJ scoring system (JAMRIS-SIJ) as an outcome measure for the quantification of interval changes in the SIJ of children and adolescents with JIA in effectiveness trials. The components of this newly developed scoring system should identify and define the measured construct, and they need not correlate with each other. Thus, the conceptual framework of the construct and measurement components are in keeping with a formative model ${ }^{13}$. This model together with the OMERACT Filter $2.0^{14,15}$ shall guide the instrument's validation framework and specify the use and interpretation of the JAMRIS-SIJ in the context of other concurrently used outcome measures in clinical trials.

Measurement domains of the OMERACT JAMRIS-SIJ. After a working group discussion and review of the existing literature, joint inflammation and structural change were selected because they measured/evaluated separate measurement constructs for the assessment of active and chronic SIJ disease in JIA. This separation is in keeping with known pathophysiology and disease history and follows from the domains in adult SIJ and other inflammatory arthritis scoring systems that have shown to be valid and feasible in the adult population ${ }^{7,8}$

Developing definitions. An anonymous survey (https://goo.gl/forms/ efnV5bs2Wpv62x132) containing multiple choice, dichotomous, and open-ended questions was developed to select the constructs, component definitions, MRI data acquisition protocol, and scoring method of an ideal pediatric SIJ MRI scoring system. The survey questions and component definitions were developed based on published adult SIJ MRI scoring systems ${ }^{8,9}$. Respondents provided suggestions to enrich the components and component definitions template, applying relevant content expertise $^{16}$.

The survey response was analyzed and presented to the OMERACT JAMRI Special Interest Group meeting during the Radiological Society of North America scientific conference in November 2017 in Chicago, Illinois, USA, for refinement of definitions and consensus upon final definitions. In May 2018, the preliminary JAMRIS-SIJ was presented to a broader group of experts at the OMERACT meeting in Terrigal, Australia, for additional input. In addition, a Steering Committee constituted of members from the adult SIJ study group was formed to align pediatric definitions with existing definitions for the adult OMERACT SIJ MRI scale. After 2 iterations of video conferences held in August and September 2018, final deliberations and consensus of items and definitions of the JAMRIS-SIJ were reached. Each item definition was voted for agreement among the members of OMERACT JAMRI Working Group as summarized in Table 1. A consensus was deemed to have been achieved if $>70 \%$ agreement was reached among

Personal non-commercial use only. The Journal of Rheumatology Copyright $\subset$ (2019. All rights reserved. 
Table 1. Consensus definitions of components of the JAMRIS-SIJ.

\begin{tabular}{ll}
\hline Feature & Definition \\
\hline $\begin{array}{l}\text { Inflammation MRI components } \\
\text { BME }\end{array}$ & $\begin{array}{l}\text { An ill-defined area of high bone marrow signal intensity } \dagger \text { on fluid-sensitive sequences within the } \\
\text { subchondral bone of the ilium or sacrum } \\
\text { Increased signal intensity on fluid sensitive and/or contrast-enhanced T1-weighted images within the } \\
\text { joint space of the cartilaginous portion of the SIJ } \\
\text { Increased signal intensity on fluid sensitive and/or contrast-enhanced T1-weighted images involving the } \\
\text { SIJ capsule } \\
\text { Increased signal intensity within the bone marrow and/or adjacent soft tissue on fluid sensitive and/or } \\
\text { contrast-enhanced T1-weighted images at sites where ligaments and tendons attach to a bone }\end{array}$ \\
Enthesitis & $\begin{array}{l}\text { A substantially wider than normal (of } \geq 5 \text { mm in adolescents) area of low subarticular bone signal } \\
\text { on T1-weighted and fluid sensitive images } \\
\text { Bony defect (or irregularity with associated BME, sclerosis, or fatty lesion) at the osteochondral } \\
\text { interface involving the bone contour and presenting with low signal intensity on T1-weighted and } \\
\text { fluid-sensitive images } \\
\text { Homogeneous increased signal intensity within the subchondral bone on T1-weighted non-FS image } \\
\text { presenting with a distinct border of the lesion } \\
\text { Presence of signal equivalent to regional bone marrow continuously bridging a portion of the joint space } \\
\text { between the iliac and sacral bones }\end{array}$ \\
Frosion &
\end{tabular}

Statement of overarching consideration for all definitions: "[...] in comparison to physiological changes normally seen on MRI examinations of age- and sex-matched children, and visible in 2 planes wherever available." $\dagger$ Caveat for BME: "[...] compared to the signal intensity of the iliac crest, edges of the vertebrae, and triradiate cartilage wherever available.” JAMRIS-SIJ: Juvenile Arthritis Magnetic Resonance Image Sacroiliac Joint Scoring System; MRI: magnetic resonance imaging; BME: bone marrow edema; SIJ: sacroiliac joint; FS: fat suppressed.

voting attendees at the session in the absence of $>15 \%$ present or more in strong disagreement ${ }^{15}$. The final results were then presented to the entire OMERACT JAMRI SIJ Working Group for endorsement. A summary of the consensus framework is presented in Appendix 1.

The study was approved by the Sickkids Research Ethics Board, REB Number 1000059077, and participation was voluntary with implied consent.

\section{RESULTS}

Our study reported an all-inclusive definition of SIJ MRI lesion components without the use of contrast for acquisition of MRI sequences. This allows for the avoidance of unnecessary use of contrast in pediatric imaging because of concerns of potential brain accumulations and nephrogenic systemic fibrosis ${ }^{17}$. Twenty-eight international multidisciplinary experts from North America $(\mathrm{n}=15)$, Europe $(\mathrm{n}=10)$, South Asia $(\mathrm{n}=2)$, and South America $(\mathrm{n}=1)$ participated in the study. The SickKids Research Ethics Board approved the use of anonymized images for the surveys, and research agreements were made among collaborating centers. Participating experts voluntarily elected to collaborate in the study.

The consensus items and definitions of the OMERACT JAMRIS-SIJ are presented in Table 1, with representative SIJ MR images in Figure 1 and Figure 2. Joint inflammation and structural changes are constructs defined separately on SIJ MRI acquired using a dedicated acquisition protocol and defined according to the components. To reiterate the complexities and pitfalls of interpreting the growing/maturing SIJ, the group decided to include a statement of "overarching consideration" for all definitions and a caveat for the inter- pretation of bone marrow edema in Table 1 . These contextual considerations require pediatric-specific experience in image interpretation. The working group acknowledges that cartilage and bone marrow development are an ongoing process in children, resulting in changes that occur because of physiologic maturity unique to sex and age 18,19,20. $^{2}$. Transformation between hematopoietic and fatty marrow occurs with advancing age, with earlier skeletal maturation noted in girls than in boys ${ }^{19,21}$.

\section{DISCUSSION}

Our study aimed at facilitating the development of a pediatric-specific MRI scoring system for measuring disease activity and structural joint changes in the SIJ of JIA. The study identified components that encompass both constructs, active joint inflammation and structural joint changes in JIA, through an iterative consensus process that allowed for both anonymized and in-person discussions and consensus quantification. The experts agreed that the preliminary JAMRIS-SIJ definitions have an acceptable face and content validity, allowing for a comprehensive measurement of the underlying construct ${ }^{14}$. Data-driven assessment of the lesion definitions is to follow.

However, as stated above, the aim of this process is to develop an outcome measure, not a diagnostic tool. The working group noted that there is a need to define an internal comparator to differentiate variations because of MRI acquisition and inter-subject variation. These can be used whenever available as normal references for bone marrow 

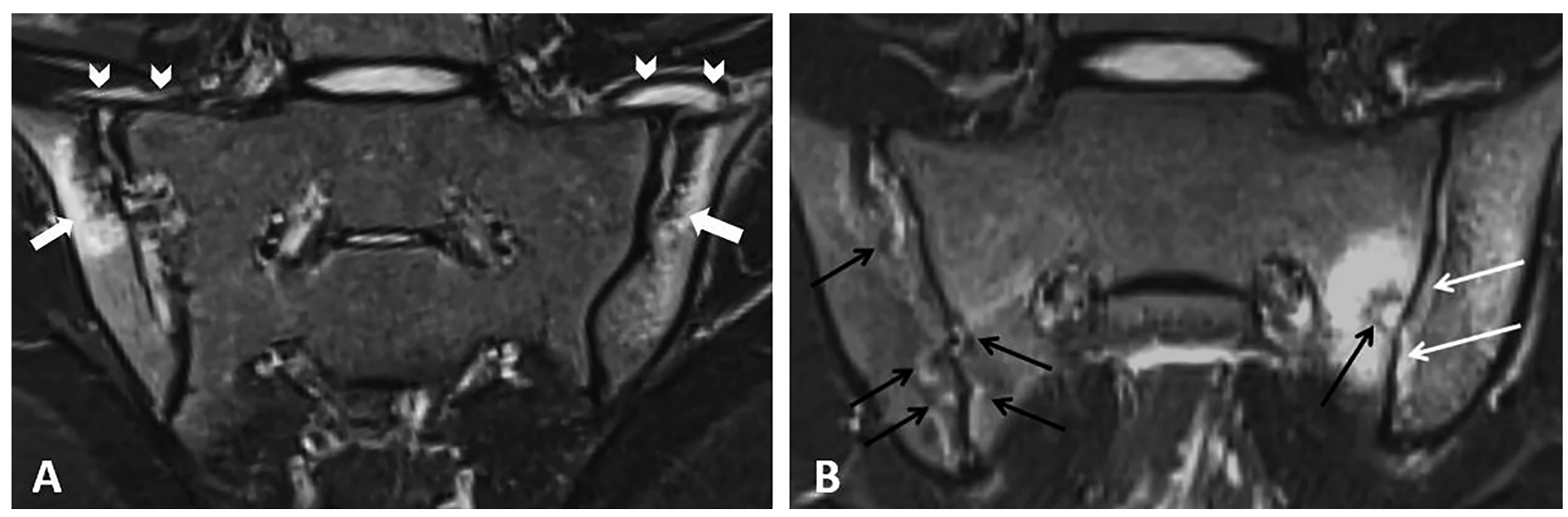

Figure 1. Coronal oblique T2-STIR MR images through the sacroiliac joints (SIJ) illustrate inflammatory components. A. SIJ in a 16-year-old girl with juvenile idiopathic arthritis (JIA) demonstrates bone marrow edema as subchondral marrow high signal intensity (arrows) along the iliac articular surfaces and capsulitis as hyperintense signal intensity in the antero-superior joint capsule (arrowheads), more prominent on the left side. B. SIJ in a 16-year-old boy with JIA shows joint space inflammation as increased signal intensity within the joint space of the cartilaginous portion of the left SI joint (white arrows) and erosion as a bony defect at the osteochondral interface (black arrows) bilaterally with increased marrow signal surrounding the left-sided erosion. STIR: short-tau inversion recovery; MR: magnetic resonance.
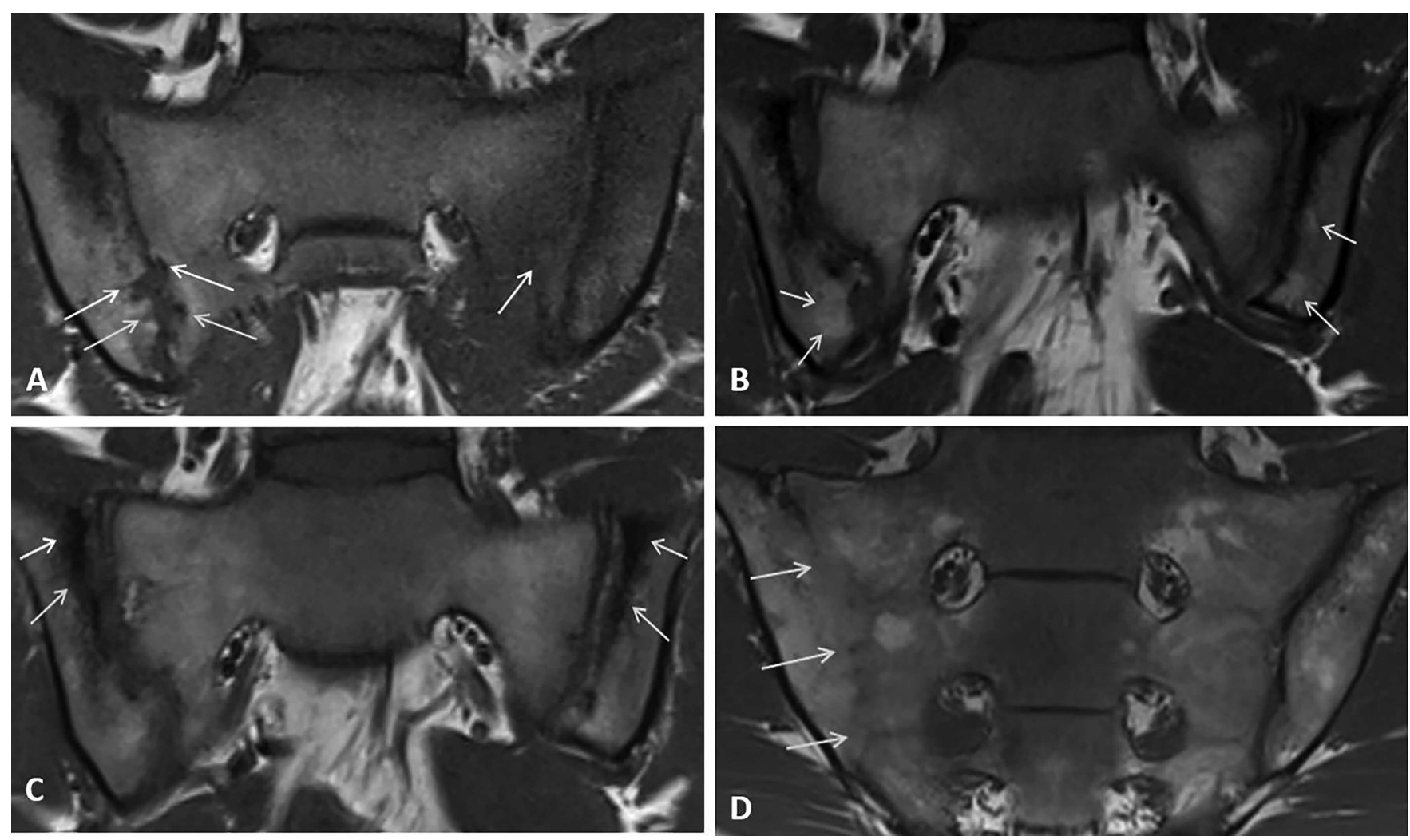

Figure 2. Coronal oblique T1-weighted MR images through the SIJ illustrate structural components. A. SIJ in a 16-year-old girl with JIA demonstrates erosions as bony defects at the osteochondral interface (arrows) bilaterally, with the left-sided sacral erosion surrounded by increased bone marrow signal intensity on fluid-sensitive images (not shown) representing bone marrow edema. B. SIJ in a 16-year-old girl with JIA shows subchondral fatty lesions as areas of homogeneous increased periarticular marrow signal intensity (arrows). C. SIJ in a 16-year-old girl with JIA depicts sclerosis as an area of very low subarticular bone marrow signal (arrows). D. SIJ in an 18-year-old boy with JIA shows ankylosis on the right side as continuous bone marrow signal that bridges the joint space and obliterates the articular surfaces of the SIJ (arrows). MR: magnetic resonance; SIJ: sacroiliac joints; JIA: juvenile idiopathic arthritis.

signal: the sacral interforaminal area, the iliac crest, ischial pubic synchondrosis, and triradiate cartilage ${ }^{11}$.

Notwithstanding the consensus obtained for lesion defini- tions among the expert group, there were areas of disagreement. The definition of erosion remains a contentious topic, because erosions may be solitary or in association with

$$
\text { Personal non-commercial use only. The Journal of Rheumatology Copyright @ } 2019 \text {. All rights reserved. }
$$


other lesions. Also, topics such as presence of erosive changes in association with differences in skeletal maturation according to sex and other common pitfalls should be reconsidered by the expert group in the future to improve the usability of the JAMRIS-SIJ. The development of an atlas for MRI of SIJ in the future should enable definition of thresholds between normality and abnormality of SIJ in the pediatric population. Further studies are required to define the minimum, ideal, and recommended data acquisition protocol, measurement scale, and methods for the OMERACT JAMRIS-SIJ.

We have reported the lesion component definitions for a preliminary pediatric MRI SIJ scoring system according to OMERACT standards - the OMERACT JAMRIS-SIJ for the assessment of inflammatory and structural joint changes in JIA. The results of this consensus-driven methodologic and technical survey conducted among a multidisciplinary international expert group are the first steps toward the development of the OMERACT JAMRIS-SIJ scoring system. Further assessment is needed, including derivation of lesion scoring method and component score, determination of MRI data acquisition protocol, exercises to systematically evaluate reliability, validity, responsiveness, and feasibility of the lesion definitions.

\section{ACKNOWLEDGMENT}

The authors acknowledge the Hospital for SickKids Research Trainee competition (RESTRACOMP) for funding provided to Dr. Tarimobo M. Otobo, and the UK National Institute for Health Research Leeds Biomedical Research Centre for funding provided to Philip G. Conaghan. The authors also acknowledge Dr. Tore A. Larheim for participating in a consensus meeting.

\section{REFERENCES}

1. Cavallo S, Brosseau L, Toupin-April K, Wells GA, Smith CA, Pugh AG, et al. Ottawa panel evidence-based clinical practice guidelines for structured physical activity in the management of juvenile idiopathic arthritis. Arch Phys Med Rehabil 2017;98:1018-41.

2. Manners PJ, Bower C. Worldwide prevalence of juvenile arthritis why does it vary so much? J Rheumatol 2002;29:1520-30.

3. Jaremko JL, Liu L, Winn NJ, Ellsworth JE, Lambert RG. Diagnostic utility of magnetic resonance imaging and radiography in juvenile spondyloarthritis: evaluation of the sacroiliac joints in controls and affected subjects. J Rheumatol 2014;41:963-70.

4. Telli H, Telli S, Topal M. The validity and reliability of provocation tests in the diagnosis of sacroiliac joint dysfunction. Pain Physician 2018;21:E367-e76.

5. Colebatch-Bourn AN, Edwards CJ, Collado P, D'Agostino MA, Hemke R, Jousse-Joulin S, et al. EULAR-PReS points to consider for the use of imaging in the diagnosis and management of juvenile idiopathic arthritis in clinical practice. Ann Rheum Dis 2015;74:1946-57.
6. Swami VG, Jaremko JL, Rumsey DG, Stimec J, Tse SML, Otobo TM, et al. Diagnostic accuracy of MRI-based sacroiliitis scoring systems: a systematic review. AJR Am J Roentgenol 2019;212: 1112-25.

7. Maksymowych WP, Wichuk S, Chiowchanwisawakit P, Lambert RG, Pedersen SJ. Development and preliminary validation of the spondyloarthritis research consortium of Canada magnetic resonance imaging sacroiliac joint structural score. J Rheumatol 2015;42:79-86.

8. Maksymowych WP, Inman RD, Salonen D, Dhillon SS, Williams M, Stone M, et al. Spondyloarthritis research Consortium of Canada magnetic resonance imaging index for assessment of sacroiliac joint inflammation in ankylosing spondylitis. Arthritis Rheum 2005;53:703-9.

9. Rudwaleit M, Jurik AG, Hermann KG, Landewe R, van der Heijde $\mathrm{D}$, Baraliakos X, et al. Defining active sacroiliitis on magnetic resonance imaging (MRI) for classification of axial spondyloarthritis: a consensual approach by the ASAS/OMERACT MRI group. Ann Rheum Dis 2009;68:1520-7.

10. Lambert RG, Bakker PA, van der Heijde D, Weber U, Rudwaleit M, Hermann KG, et al. Defining active sacroiliitis on MRI for classification of axial spondyloarthritis: update by the ASAS MRI working group. Ann Rheum Dis 2016;75:1958-63.

11. Weiss PF, Maksymowych WP, Lambert RG, Jaremko JL, Biko DM, Paschke J, et al. Feasibility and reliability of the Spondyloarthritis Research Consortium of Canada sacroiliac joint inflammation score in children. Arthritis Res Ther 2018;20:56.

12. Weiss PF, Maksymowych WP, Lambert RG, Jaremko JL, Biko DM, Paschke J, et al. Feasibility and reliability of the Spondyloarthritis Research Consortium of Canada sacroiliac joint structural score in children. J Rheumatol 2018;45:1411-7.

13. Edwards JR, Bagozzi RP. On the nature and direction of relationships between constructs and measures. Psychol Methods 2000;5:155-74

14. Boers M, Kirwan JR, Wells G, Beaton D, Gossec L, d'Agostino MA, et al. Developing core outcome measurement sets for clinical trials: OMERACT filter 2.0. J Clin Epidemiol 2014;67:745-53.

15. Boers M, Kirwan J, Tugwell P, Beaton D, Bingham CI, Conaghan P. The OMERACT Handbook. [Internet. Accessed March 21, 2019.] Available from: https://omeract.org/resources

16. Feinstein AR. Clinimetric perspectives. J Chronic Dis 1987; 40:635-40.

17. Ranga A, Agarwal Y, Garg KJ. Gadolinium based contrast agents in current practice: risks of accumulation and toxicity in patients with normal renal function. Indian J Radiol Imaging 2017;27:141-7.

18. Navallas M, Ares J, Beltrán B, Lisbona MP, Maymó J, Solano A. Sacroiliitis associated with axial spondyloarthropathy: new concepts and latest trends. Radiographics 2013;33:933-56.

19. Bray TJ, Vendhan K, Roberts J, Atkinson D, Punwani S, Sen D, et al. Association of the apparent diffusion coefficient with maturity in adolescent sacroiliac joints. J Magn Reson Imaging 2016; 44:556-64.

20. Herregods N, Dehoorne J, Van den Bosch F, Jaremko JL, Van Vlaenderen J, Joos R, et al. ASAS definition for sacroiliitis on MRI in SpA: applicable to children? Pediatr Rheumatol Online J 2017;15:24.

21. Laor T, Jaramillo D. MR imaging insights into skeletal maturation: what is normal? Radiology 2009;250:28-38. 
APPENDIX 1. Summary of the methodology and consensus framework for the development of an MRI-based scoring system including its constructs, components, and the consensus process for component definition. MRI: magnetic resonance imaging; SIJ: sacroiliac joint; BME: bone marrow edema; JAMRIS-SIJ: Juvenile Arthritis MRI SIJ scoring system.

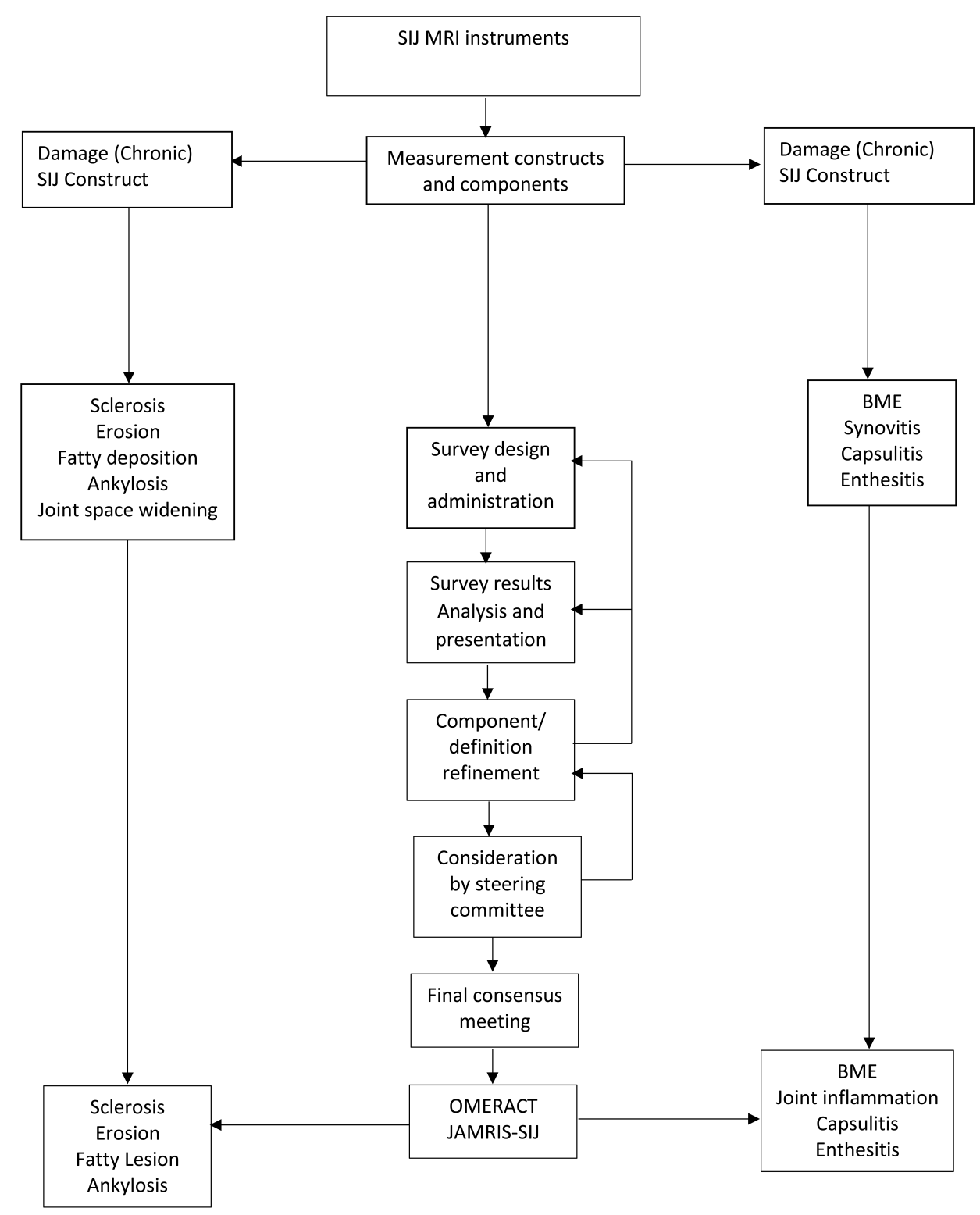

